\title{
On Bihermitian Matrices
}

\author{
G.Ramesh*, N.Anbarasi** \\ *Associate Professor of Mathematics, Govt. Arts College(Autonomous), Kumbakonam. \\ **Assistant Professor of Mathematics, Arasu Engineering College, Kumbakonam.
}

Abstract: Bihermitian matrices are studied as a generalization of hermitian matrices. Some of the properties of hermitian matrices are extended to bihermitian matrices. Some important results of hermitian matrices are generalized to bihermitian matrices.

Keywords: Hermitian matrix, skew-hermitian matrix, bimatrix, bihermitian matrix, skew bihermitian matrix. AMS Classification: 15A09, 15A15, $15 \mathrm{~A} 57$.

\section{Introduction}

Matrices provide a very powerful tool for dealing with linear models. Bimatrices are still a powerful and an advanced tool which can handle over one linear model at a time. Bimatrices are useful when time bound comparisons are needed in the analysis of a model. Bimatrices are of several types. Here we consider all matrices belongs to $C_{n x n}$. For any matrix A, $A^{H}$ denotes the conjugate transpose of A. In this paper we study bihermitian matrices as a generalization of hermitian matrices. Some of the properties of hermitian matrices are extended to bihermitian matrices. Some important results of hermitian matrices are generalized to bihermitian matrices.

Definition 2.1[3]

\section{Preliminaries}

A bimatrix $A_{B}$ is defined as the union of two rectangular array of numbers $A_{1}$ and $A_{2}$ arranged into rows and columns. It is written as $A_{B}=A_{1} \cup A_{2}$ with $A_{1} \neq A_{2}$ (except zero and unit bimatrices) where,

$$
\mathrm{A}_{1}=\left[\begin{array}{cccc}
a_{11}^{1} & a_{12}^{1} & \cdots & a_{1 n}^{1} \\
a_{21}^{1} & a_{22}^{1} & \cdots & a_{2 n}^{1} \\
\vdots & & & \\
a_{m 1}^{1} & a_{m 2}^{1} & \cdots & a_{m n}^{1}
\end{array}\right] \text { and } \mathrm{A}_{2}=\left[\begin{array}{cccc}
a_{11}^{2} & a_{12}^{2} & \cdots & a_{1 n}^{2} \\
a_{21}^{2} & a_{22}^{2} & \cdots & a_{2 n}^{2} \\
\vdots & & & \\
a_{m 1}^{2} & a_{m 2}^{2} & \cdots & a_{m n}^{2}
\end{array}\right]
$$

' $\cup$ ' is just the notational convenience (symbol) only.

Definition 2.2[3]

Let $A_{B}=A_{1} \cup \mathrm{A}_{2}$ and $C_{B}=C_{1} \cup C_{2}$ be any two mxn bimatrices. The sum $D_{B}$ of the bimatrices $A_{B}$ and $C_{B}$ is defined as

$D_{B}=A_{B}+C_{B}=\left(A_{1} \cup \mathrm{A}_{2}\right)+\left(C_{1} \cup C_{2}\right)=\left(\mathrm{A}_{1}+C_{1}\right) \cup\left(A_{2}+C_{2}\right)$, where $A_{1}+A_{2}$ and $C_{1}+C_{2}$ are the usual addition of matrices.

Definition 2.3[4]

If $A_{B}=A_{1} \cup A_{2}$ and $C_{B}=C_{1} \cup C_{2}$ be two bimatrices, then $A_{B}$ and $C_{B}$ are said to be equal (written as $A_{B}=C_{B}$ ) if and only if $A_{1}$ and $C_{1}$ are identical and $A_{2}$ and $C_{2}$ are identical. (That is, $A_{1}=C_{1}$ and $A_{2}=C_{2}$ ).

Definition 2.4[4]

Given a bimatrix $A_{B}=A_{1} \cup \mathrm{A}_{2}$ and a scalar $\lambda$, the product of $\lambda$ and $A_{B}$ written as $\lambda A_{B}$ is defined to be 


$$
\lambda A_{B}=\left[\begin{array}{cccc}
\lambda a_{11}^{1} & \lambda a_{12}^{1} & \cdots & \lambda a_{1 n}^{1} \\
\lambda a_{21}^{1} & \lambda a_{22}^{1} & \cdots & \lambda a_{2 n}^{1} \\
\vdots & & & \\
\lambda a_{m 1}^{1} & \lambda a_{m 2}^{1} & \cdots & \lambda a_{m n}^{1}
\end{array}\right] \cup\left[\begin{array}{cccc}
\lambda a_{11}^{2} & \lambda a_{12}^{2} & \cdots & \lambda a_{1 n}^{2} \\
\lambda a_{21}^{2} & \lambda a_{22}^{2} & \cdots & \lambda a_{2 n}^{2} \\
\vdots & & & \\
\lambda a_{m 1}^{2} & \lambda a_{m 2}^{2} & \cdots & \lambda a_{m n}^{2}
\end{array}\right]
$$

That is, each element of $A_{1}$ and $A_{2}$ are multiplied by $\lambda$.

\section{Remark 2.5[4]}

If $A_{B}=A_{1} \cup A_{2}$ be a bimatrix, then we call $A_{1}$ and $A_{2}$ as the component matrices of the bimatrix $A_{B}$.

\section{Definition 2.6[3]}

If $A_{B}=A_{1} \cup \mathrm{A}_{2}$ and $C_{B}=C_{1} \cup C_{2}$ are both nxn square bimatrices then, the bimatrix multiplication is defined as,

$$
\mathrm{A}_{B} \times C_{B}=\left(A_{1} C_{1}\right) \cup\left(A_{2} C_{2}\right) .
$$

\section{Definition 3.1}

\section{Hermitian Bimatrices}

A bihermitian matrix is a bimatrix $A_{B}=A_{1} \cup \mathrm{A}_{2}$ for which $A_{B}=A_{B}^{H}$. That is, the component matrices of $A_{B}$ are hermitian matrices. (That is, $A_{B}=A_{B}^{H}=A_{1}^{H} \cup A_{2}^{H}$ ).

\section{Remark 3.2}

Let $A_{B}=A_{1} \cup \mathrm{A}_{2}$ be a bihermitian matrix. If $\mathrm{A}_{1}$ and $\mathrm{A}_{2}$ are square and posses the same order then $A_{B}$ is called square bihermitian matrix, and if $A_{1}$ and $A_{2}$ are of different orders then $A_{B}$ is called mixed square bihermitian matrix.

\section{Example 3.3}

$$
(i) A_{B}=\left[\begin{array}{ccc}
1 & 1-i & -3+2 i \\
1+i & 2 & -i \\
-3-2 i & i & 0
\end{array}\right] \cup\left[\begin{array}{ccc}
1 & 1+2 i & 2-3 i \\
1-2 i & 5 & -4-2 i \\
2+3 i & -4+2 i & 13
\end{array}\right]
$$

is a square bihermitian.

$$
\text { (ii) } A_{B}=\left[\begin{array}{lcc}
3 & 2-i & -3 i \\
2+i & 0 & 1-i \\
3 i & 1+i & 0
\end{array}\right] \cup\left[\begin{array}{cc}
2 & 2+i \\
2-i & 3
\end{array}\right]
$$

\section{Remark 3.4}

is a mixed square bihermitian.

Any complex matrix can be represented as $A=A_{R}+i A_{I}$, where $A_{R}$ is symmetric matrix and $A_{I}$ is skew symmetric matrix. Now, any bihermitian matrix $A_{B}=A_{1} \cup \mathrm{A}_{2}$ can be represented as,

$$
A_{B}=\left(A_{1 R}+i A_{1 I}\right) \cup\left(A_{2 R}+i A_{2 I}\right)=\left(A_{1 R}^{T}-i A_{1 I}^{T}\right) \cup\left(A_{2 R}^{T}-i A_{2 I}^{T}\right)=A_{1}^{H} \cup A_{2}^{H}=A_{B}^{H}
$$

\section{Definition 3.5}

Let $\mathrm{A}_{B}=\mathrm{A}_{1} \cup A_{2}$ be a nxn square bimatrix. That is, $A_{1}$ and $A_{2}$ are nxn square matrices. A skew hermitian bimatrix is a bimatrix $A_{B}$ for which $A_{B}=-A_{B}^{H}$, where $-A_{B}^{H}=-A_{1}^{H} \cup-A_{2}^{H}$. That is, the component matrices $A_{1}$ and $A_{2}$ of $A_{B}$ are skew hermitian matrices.

\section{Theorem 3.6}

If $A_{B}$ and $B_{B}$ are bihermitian matrices then $\left(A_{B}+B_{B}\right)$ is also bihermitian.

Proof

Given $A_{B}=A_{1} \cup \mathrm{A}_{2}$ and $B_{B}=B_{1} \cup \mathrm{B}_{2}$ are bihermitian. 


$$
\begin{aligned}
& \begin{aligned}
\left(\mathrm{A}_{\mathrm{B}}+B_{B}\right)^{H} & =A_{B}^{H}+B_{B}^{H} \\
& =\left(A_{1} \cup A_{2}\right)^{\mathrm{H}}+\left(B_{1} \cup \mathrm{B}_{2}\right)^{\mathrm{H}} \\
= & \left(\mathrm{A}_{1}^{\mathrm{H}} \cup A_{2}^{H}\right)+\left(B_{1}^{H} \cup B_{2}^{H}\right) \\
= & \left(A_{1} \cup A_{2}\right)+\left(B_{1} \cup \mathrm{B}_{2}\right) \\
\qquad & \left(\sin c e A_{1}, A_{2}, B_{1} \text { and } B_{2} \text { are Hermitian matrices }\right)
\end{aligned} \\
& \left(A_{B}+B_{B}\right)^{H}=A_{B}+B_{B} \\
& \text { Hence, }\left(A_{B}+B_{B}\right) \text { is bihermitian. }
\end{aligned}
$$

Example 3.7

$$
\begin{aligned}
& \text { Let } A_{B}=\left[\begin{array}{ccc}
1 & 1-i & -3+2 i \\
1+i & 2 & -i \\
-3-2 i & i & 0
\end{array}\right] \cup\left[\begin{array}{ccc}
1 & 1+2 i & 2-3 i \\
1-2 i & 5 & -4-2 i \\
2+3 i & -4+2 i & 13
\end{array}\right] \\
& \text { and } B_{\mathrm{B}}=\left[\begin{array}{llr}
1 & -2 i & 0 \\
2 i & -1 & 2 i \\
0 & -2 i & 2
\end{array}\right] \cup\left[\begin{array}{ccc}
1 & 2 i & -i \\
-2 i & -1 & 1 \\
i & 1 & 2
\end{array}\right] \\
& A_{B}+B_{B}=\left[\begin{array}{ccc}
2 & 1-3 i & -3+2 i \\
1+3 i & 1 & i \\
-3-2 i & i & 2
\end{array}\right] \cup\left[\begin{array}{ccc}
1 & 2 i & -i \\
-2 i & -1 & 1 \\
-i & 1 & 2
\end{array}\right]
\end{aligned}
$$

Hence, $A_{B}+B_{B}$ is bihermitian.

\section{Theorem 3.8}

If $A_{B}$ is bihermitian, for any scalar $k,\left(\mathrm{~A}_{\mathrm{B}}-k I_{B}\right)$ is bihermitian.

\section{Proof}

$$
\text { Given } A_{B}=A_{1} \cup A_{2}=A_{1}^{H} \cup A_{2}^{H}
$$

That is, $A_{B}=\left(A_{1 R}+i A_{1 I}\right) \cup\left(A_{2 R}+i A_{2 I}\right)=\left(A_{1 R}^{T}-i A_{1 I}^{T}\right) \cup\left(A_{2 R}^{T}-i A_{2 I}^{T}\right)$

$$
\begin{aligned}
\left(A_{B}-k I_{B}\right)^{H} & =\left[\left(A_{1} \cup A_{2}\right)-k\left(I_{1} \cup I_{2}\right)\right]^{H} \\
& =\left[\left(A_{1} \cup A_{2}\right)-\left(k I_{1} \cup k I_{2}\right)\right]^{H} \\
& =\left[\left(A_{1}-k I_{1}\right) \cup\left(A_{2}-k I_{2}\right)\right]^{H} \\
& =\left[\left(A_{1 R}+i A_{1 I}\right)-k I_{1}\right]^{H} \cup\left[\left(A_{2 R}+i A_{2 I}\right)-k I_{2}\right]^{H} \\
& =\left[\left(A_{1 R}-k I_{1}\right)+i A_{1 I}\right]^{H} \cup\left[\left(A_{2 R}-k I_{2}\right)+i A_{2 I}\right]^{H} \\
& =\left[\left(A_{1 R}-k I_{1}\right)^{T}-i A_{1 I}^{T}\right] \cup\left[\left(A_{2 R}-k I_{2}\right)^{T}-i A_{2 I}^{T}\right] \\
& =\left(A_{1 R}^{T}-k I_{1}^{T}-i A_{1 I}^{T}\right) \cup\left(A_{2 R}^{T}-k I_{2}^{T}-i A_{2 I}^{T}\right) \\
& =\left(A_{1 R}^{T}-i A_{1 I}^{T}\right)-k I_{1}^{T} \cup\left(A_{2 R}^{T}-i A_{2 I}^{T}\right)-k I_{2}^{T} \\
& =\left(A_{1 R}+i A_{1 I}\right)-k I_{1} \cup\left(A_{2 R}+i A_{2 I}\right)-k I_{2} \\
& =\left(A_{1}-k I_{1}\right) \cup\left(A_{2}-k I_{2}\right) \\
& =\left(A_{1} \cup A_{2}\right)-k\left(I_{1} \cup I_{2}\right)
\end{aligned}
$$




$$
=A_{B}-k I_{B}
$$

Hence, $A_{B}-k I_{B}$ is bihermitian.

\section{Example 3.9}

Consider the bihermitian matrix,

$$
\begin{aligned}
& A_{B}=\left[\begin{array}{ccc}
1 & 1-i & -3+2 i \\
1+i & 2 & -i \\
-3-2 i & i & 0
\end{array}\right] \cup\left[\begin{array}{ccc}
1 & 1+2 i & 2-3 i \\
1-2 i & 5 & -4-2 i \\
2+3 i & -4+2 i & 13
\end{array}\right] \\
& I_{B}=\left[\begin{array}{lll}
1 & 0 & 0 \\
0 & 1 & 0 \\
0 & 0 & 1
\end{array}\right] \cup\left[\begin{array}{lll}
1 & 0 & 0 \\
0 & 1 & 0 \\
0 & 0 & 1
\end{array}\right] \\
& k I_{B}=\left[\begin{array}{ccc}
k & 0 & 0 \\
0 & k & 0 \\
0 & 0 & k
\end{array}\right] \cup\left[\begin{array}{ccc}
k & 0 & 0 \\
0 & k & 0 \\
0 & 0 & k
\end{array}\right] \\
& A_{B}-k I_{B}=\left[\begin{array}{ccc}
1 & 1-i & -3+2 i \\
1+i & 2 & -i \\
-3-2 i & i & 0
\end{array}\right] \cup\left[\begin{array}{ccc}
1 & 1+2 i & 2-3 i \\
1-2 i & 5 & -4-2 i \\
2+3 i & -4+2 i & 13
\end{array}\right]- \\
& {\left[\begin{array}{ccc}
k & 0 & 0 \\
0 & k & 0 \\
0 & 0 & k
\end{array}\right] \cup\left[\begin{array}{ccc}
k & 0 & 0 \\
0 & k & 0 \\
0 & 0 & k
\end{array}\right]} \\
& A_{B}-k I_{B}=\left[\begin{array}{lcc}
1-k & 1-i & -3+2 i \\
1+i & 2-k & -i \\
-3-2 i & i & -k
\end{array}\right] \cup\left[\begin{array}{ccc}
1-k & 1+2 i & 2-3 i \\
1-2 i & 5-k & -4-2 i \\
2+3 i & -4+2 i & 13-k
\end{array}\right]
\end{aligned}
$$

Which is bihermitian.

\section{Theorem 3.10}

\section{Proof}

Any integral power of a bihermitian matrix is also bihermitian.

$$
\begin{aligned}
\text { Let } & A_{B}=A_{1} \cup \mathrm{A}_{2} \text { be bihermitian. } \\
A_{B}^{H}=A_{1}^{H} \cup A_{2}^{H} & =A_{1} \cup A_{2}=A_{B} \\
\left(\mathrm{~A}_{\mathrm{B}}^{2}\right)^{H} & =\left(A_{B} A_{B}\right)^{H} \\
& =A_{B}^{H} A_{B}^{H} \\
& =\left(A_{1} \cup A_{2}\right)^{H}\left(A_{1} \cup A_{2}\right)^{H} \\
& =\left(A_{1}^{\mathrm{H}} \cup A_{2}^{H}\right)\left(A_{1}^{H} \cup A_{2}^{H}\right) \\
& =\left(A_{1}^{H}\right)^{2} \cup\left(A_{2}^{H}\right)^{2} \\
& =A_{1}^{2} \cup A_{2}^{2}\left(\sin c e A_{1} \text { and } A_{2} \text { are hermitian matrices }\right) \\
\left(A_{B}^{2}\right)^{H} & =A_{B}^{2}
\end{aligned}
$$

Hence, $A_{B}^{2}$ is bihermitian. 
Assume that $A_{B}^{k}$ is bihermitian, that is $\left(A_{B}^{k}\right)^{H}=A_{B}^{k}$

To prove $\mathrm{A}_{B}^{k+1}$ is bihermitian.

$$
\begin{aligned}
\left(\mathrm{A}_{B}^{k+1}\right)^{\mathrm{H}} & =\left(A_{B}^{k} A_{B}\right)^{\mathrm{H}} \\
& =A_{B}^{H}\left(A_{B}^{k}\right)^{H} \\
& =A_{B} A_{B}^{k} \quad(\text { since by (1) and (2)) } \\
& =A_{B}^{1+k} \\
\left(A_{B}^{k+1}\right)^{\mathrm{H}} & =A_{B}^{k+1}
\end{aligned}
$$

Hence, any integral power of a bihermitian matrix is also bihermitian.

\section{Example 3.11}

$$
\begin{aligned}
& \text { Let } A_{B}=\left[\begin{array}{lcr}
1 & 1-i & -3+2 i \\
1+i & 2 & -i \\
-3-2 i & i & 0
\end{array}\right] \cup\left[\begin{array}{ccr}
1 & 1+2 i & 2-3 i \\
1-2 i & 5 & -4-2 i \\
2+3 i & -4+2 i & 13
\end{array}\right] \\
& A_{B}{ }^{2}=\left[\begin{array}{ccc}
1 & 1-i & -3+2 i \\
1+i & 2 & -i \\
-3-2 i & i & 0
\end{array}\right] \cup\left[\begin{array}{ccc}
1 & 1+2 i & 2-3 i \\
1-2 i & 5 & -4-2 i \\
2+3 i & -4+2 i & 13
\end{array}\right] \times \\
& {\left[\begin{array}{ccc}
1 & 1-i & -3+2 i \\
1+i & 2 & -i \\
-3-2 i & i & 0
\end{array}\right] \cup\left[\begin{array}{ccc}
1 & 1+2 i & 2-3 i \\
1-2 i & 5 & -4-2 i \\
2+3 i & -4+2 i & 13
\end{array}\right]} \\
& =\left[\begin{array}{ccc}
16 & 1-6 i & -4+i \\
1+6 i & 3 & -5-3 i \\
-4-i & -5+3 i & 14
\end{array}\right] \cup\left[\begin{array}{ccc}
19 & 4+28 i & 28-52 i \\
4-28 i & 48 & -76-43 i \\
28+52 i & -76+43 i & 202
\end{array}\right]
\end{aligned}
$$

Hence, $A_{B}^{2}$ is bihermitian.

\section{Theorem 3.12}

For any square matrix $\mathrm{A}, \mathrm{A}+\mathrm{A}^{\mathrm{H}}$ is bihermitian.

\section{Proof}

Let $A_{B}$ be any square bimatrix. That is, $A_{B}=A_{1} \cup A_{2}$.

$$
\begin{aligned}
\left(A_{B}+A_{B}^{H}\right)^{H} & =\left[\left(A_{1} \cup A_{2}\right)+\left(A_{1} \cup A_{2}\right)^{H}\right]^{\mathrm{H}} \\
& =\left[\left(A_{1} \cup A_{2}\right)+\left(A_{1}^{H} \cup A_{2}^{H}\right)\right]^{H} \\
& =\left[\left(A_{1}+A_{1}^{H}\right) \cup\left(A_{2}+A_{2}^{H}\right)\right]^{H} \\
& =\left(A_{1}+A_{1}^{H}\right)^{H} \cup\left(A_{2}+A_{2}^{H}\right)^{H} \\
& =\left(A_{1}^{H}+A_{1}\right) \cup\left(A_{2}^{H}+A_{2}\right) \\
& =\left(A_{1}^{H} \cup A_{2}^{H}\right)+\left(A_{1} \cup A_{2}\right) \\
& =\left(A_{1} \cup A_{2}\right)^{H}+\left(A_{1} \cup A_{2}\right)
\end{aligned}
$$




$$
\begin{aligned}
& =A_{B}^{H}+A_{B} \\
\left(A_{B}+A_{B}^{H}\right)^{H} & =A_{B}+A_{B}^{H}
\end{aligned}
$$

Hence, $A_{B}+A_{B}{ }^{H}$ is bihermitian.

\section{Example 3.13}

$$
\text { Let } \begin{aligned}
A_{B} & =\left[\begin{array}{ccc}
1 & 2 i & i \\
0 & 2 & -i \\
0 & i & 3
\end{array}\right] \cup\left[\begin{array}{lcc}
3 & 2-i & -3 i \\
1+i & 1 & -i \\
2+i & -i & 1
\end{array}\right] \\
A_{B}^{H} & =\left[\begin{array}{ccc}
1 & 0 & 0 \\
-2 i & 2 & -i \\
-i & i & 3
\end{array}\right] \cup\left[\begin{array}{lcc}
3 & 1-i & 2-i \\
2+i & 1 & i \\
3 i & i & 1
\end{array}\right] \\
A_{B}+A_{B}^{H} & =\left[\begin{array}{ccc}
2 & 2 i & i \\
-2 i & 4 & -2 i \\
-i & 2 i & 6
\end{array}\right] \cup\left[\begin{array}{lll}
6 & 3-2 i & 2-4 i \\
3+2 i & 2 & 0 \\
2+4 i & 0 & 2
\end{array}\right] \text {, which is bihermitian . }
\end{aligned}
$$

\section{Theorem 3.14}

For any square matrix $A_{B}, A_{B}-A_{B}^{H}$ is skew bihermitian.

\section{Proof}

Let $A_{B}=A_{1} \cup A_{2}$ be a square bimatrix.

$$
\begin{aligned}
A_{B}-A_{B}^{H} & =\left(A_{1} \cup A_{2}\right)-\left(A_{1} \cup A_{2}\right)^{H} \\
& =\left(A_{1} \cup A_{2}\right)-\left(A_{1}^{H} \cup A_{2}^{H}\right) \\
A_{B}-A_{B}^{H} & =\left(A_{1}-A_{1}^{H}\right) \cup\left(A_{2}-A_{2}^{H}\right) \\
\left(A_{B}-A_{B}^{H}\right)^{\mathrm{H}} & =\left[\left(A_{1}-A_{1}^{H}\right) \cup\left(A_{2}-A_{2}^{H}\right)\right]^{H} \\
& =\left(A_{1}-A_{1}^{H}\right)^{H} \cup\left(A_{2}-A_{2}^{H}\right)^{H} \\
& =\left(A_{1}^{H}-A_{1}\right) \cup\left(A_{2}^{H}-A_{2}\right) \\
& =\left(A_{1}^{H} \cup A_{2}^{H}\right)-\left(A_{1} \cup A_{2}\right) \\
& =\left(A_{1} \cup A_{2}\right)^{H}-\left(A_{1} \cup A_{2}\right) \\
& =A_{B}^{H}-A_{B} \\
\left(A_{B}-A_{B}^{H}\right)^{\mathrm{H}}= & -\left(A_{B}-A_{B}^{H}\right)
\end{aligned}
$$

Hence, $A_{B}-A_{B}^{H}$ is skew bihermitian.

\section{Theorem 3.15}

If $A_{B}$ is bihermitian then $i A_{B}$ is skew bihermitian.

\section{Proof}

Given $A_{B}$ is bihermitian. That is, $A_{B}^{H}=A_{1}^{H} \cup A_{2}^{H}=A_{B}$

Now, $\left(i A_{B}\right)^{H}=\left[i\left(A_{1} \cup A_{2}\right)\right]^{H}$

$$
=-i\left(A_{1} \cup A_{2}\right)^{H}
$$




$$
\begin{aligned}
& =-i\left(A_{1}{ }^{H} \cup A_{2}{ }^{H}\right) \\
& =-i\left(A_{1} \cup A_{2}\right) \quad\left(\sin c e A_{1} \text { and } A_{2} \text { are hermitian }\right) \\
\left(i A_{B}\right)^{H} & =-i A_{B}
\end{aligned}
$$

Hence, $i A_{B}$ is skew bihermitian.

\section{Example 3.16}

$$
\text { Let } \begin{aligned}
A_{B} & =\left[\begin{array}{lcc}
1 & 1-i & -3+2 i \\
1+i & 2 & -i \\
-3-2 i & i & 0
\end{array}\right] \cup\left[\begin{array}{ccc}
1 & 1+2 i & 2-3 i \\
1-2 i & 5 & -4-2 i \\
2+3 i & -4+2 i & 13
\end{array}\right] \\
i A_{B}= & {\left[\begin{array}{ccc}
i & 1+i & -2-3 i \\
-1+i & 2 i & 1 \\
2-3 i & -1 & 0
\end{array}\right] \cup\left[\begin{array}{ccc}
i & -2+i & 3+2 i \\
2+i & 5 i & 2-4 i \\
-3+2 i & -2-4 i & 13 i
\end{array}\right] } \\
\left(i A_{B}\right)^{H}= & {\left[\begin{array}{lrc}
-i & -1-i & 2+3 i \\
1-i & -2 i & -1 \\
-2+3 i & 1 & 0
\end{array}\right] \cup\left[\begin{array}{ccc}
-i & 2-i & 2 i+3 \\
-2-i & -5 i & -2+4 i \\
3-2 i & 2+4 i & -13 i
\end{array}\right] } \\
= & \left.-\left\{\begin{array}{lrc}
i \\
-1 & 1+i & -2-3 i \\
-1+i & 2 i & 1 \\
2-3 i & -1 & 0
\end{array}\right] \cup\left[\begin{array}{lll}
i & -2+i & 3+2 i \\
2+i & 5 i & -2+4 i \\
-3+2 i & -2-4 i & 13 i
\end{array}\right]\right\} \\
\left(i A_{B}\right)^{H}= & -\left(i A_{B}\right)
\end{aligned}
$$

Hence, $i A_{B}$ is skew bihermitian.

\section{Theorem 3.17}

If $A_{B}$ is skew bihermitian then $i A_{B}$ is bihermitian.

Proof

Given $A_{B}$ is skew bihermitian, that is $A_{B}=-A_{B}^{H}$

$$
\begin{aligned}
\left(i A_{B}\right)^{H} & =\left[i\left(A_{B}\right)\right]^{H} \\
& =-i A_{B}{ }^{H} \\
& =-i\left(A_{1} \cup A_{2}\right)^{H} \\
& =-i\left(A_{1}{ }^{H} \cup A_{2}{ }^{H}\right) \\
& =i\left(-A_{1}{ }^{H} \cup-A_{2}{ }^{H}\right) \\
& =i\left(A_{1} \cup A_{2}\right), \quad\left(\sin c e A_{1} \text { and } A_{2} \text { are skew hermitian }\right) \\
& =i A_{B}
\end{aligned}
$$

Hence, $i A_{B}$ is bihermitian.

\section{Theorem 3.18}

Any bimatrix $A_{B}$ can be uniquely written in the form, $A_{B}=H_{B}+S_{B}$, where $H_{B}$ is bihermitian and $S_{B}$ is skew bihermitian.

\section{Proof}

Let $\mathrm{A}$ be any square matrix.

We know that any square matrix A can be represented as, 


$$
\begin{aligned}
& A=\left(\frac{A+A^{H}}{2}\right)+\left(\frac{A-A^{H}}{2}\right), \text {-------> (1) } \\
& \quad \text { where } \frac{A+A^{H}}{2} \text { is hermitian and } \frac{A-A^{H}}{2} \text { is skew hermitian. }
\end{aligned}
$$

Let $A_{B}=A_{1} \cup A_{2}$ be any square bimatrix, where $A_{1}$ and $A_{2}$ are any two square matrices.

$$
\text { Let } \begin{aligned}
A_{B} & =\frac{A_{B}+A_{B}^{H}}{2}+\frac{A_{B}-A_{B}^{H}}{2} \\
& =\frac{\left(A_{1} \cup A_{2}\right)+\left(A_{1} \cup A_{2}\right)^{H}}{2}+\frac{\left(A_{1} \cup A_{2}\right)-\left(A_{1} \cup A_{2}\right)^{H}}{2} \\
& =\frac{\left(A_{1} \cup A_{2}\right)+\left(A_{1}^{H} \cup A_{2}^{H}\right)}{2}+\frac{\left(A_{1} \cup A_{2}\right)-\left(A_{1}^{H} \cup A_{2}^{H}\right)}{2} \\
& =\frac{\left(A_{1}+A_{1}^{H}\right) \cup\left(A_{2}+A_{2}^{H}\right)}{2}+\frac{\left(A_{1}-A_{1}^{H}\right) \cup\left(A_{2}-A_{2}^{H}\right)}{2} \\
& =\left[\left(\frac{A_{1}+A_{1}^{H}}{2}\right) \cup\left(\frac{A_{2}+A_{2}^{H}}{2}\right)\right]+\left[\left(\frac{A_{1}-A_{1}^{H}}{2}\right) \cup\left(\frac{A_{2}-A_{2}^{H}}{2}\right)\right] \\
& =\left[\left(\frac{A_{1}+A_{1}^{H}}{2}\right)+\left(\frac{A_{1}-A_{1}^{H}}{2}\right)\right] \cup\left[\left(\frac{A_{2}+A_{2}^{H}}{2}\right)+\left(\frac{A_{2}-A_{2}^{H}}{2}\right)\right] \\
& =\left(H_{1}+S_{1}\right) \cup\left(H_{2}+S_{2}\right),
\end{aligned}
$$$$
\text { where } H_{1}, H_{2} \text { are hermitian and } S_{1}, S_{2} \text { are skew hermitian(since by (1)) }
$$

Hence, $A_{B}=H_{B}+S_{B}$, Where $H_{B}$ is bihermitian and $S_{B}$ is skew bihermitian.

\section{Example 3.18}

$$
\begin{aligned}
& \text { Let } A_{B}=\left[\begin{array}{llc}
2+i & 2-2 i & -5-i \\
4 i & 1+2 i & 1+i \\
-1-5 i & -1-i & 2
\end{array}\right] \cup\left[\begin{array}{lll}
1+i & -2+3 i & 3+i \\
2-i & -1+5 i & 3-4 i \\
-3+3 i & -1-4 i & 2+13 i
\end{array}\right]=A_{1} \cup A_{2} \text { (say) } \\
& \text { where } A_{1}=\left[\begin{array}{ccc}
2+i & 2-2 i & -5-i \\
4 i & 1+2 i & 1+i \\
-1-5 i & -1-i & 2
\end{array}\right] \quad ; \quad A_{1}^{H}=\left[\begin{array}{ccc}
2-i & -4 i & -1+5 i \\
2+2 i & 1-2 i & -1+i \\
-5+i & 1-i & 2
\end{array}\right] \\
& \text { and } \quad A_{2}=\left[\begin{array}{lll}
1+i & -2+3 i & 3+i \\
2-i & -1+5 i & 3-4 i \\
-3+3 i & -1-4 i & 2+13 i
\end{array}\right] ; \quad A_{2}^{H}=\left[\begin{array}{lll}
1-i & 2+i & -3-3 i \\
-2-3 i & -1-5 i & -1+4 i \\
3-i & 3+4 i & 2-13 i
\end{array}\right] \\
& \frac{A_{1}+A_{1}^{H}}{2}=\frac{1}{2}\left[\begin{array}{lcc}
4 & 2-6 i & -6+4 i \\
2+6 i & 2 & 2 i \\
-6-4 i & -2 i & 4
\end{array}\right]=\left[\begin{array}{ccc}
2 & 1-3 i & -3+2 i \\
1+3 i & 1 & i \\
-3-2 i & -i & 2
\end{array}\right]=H_{1}(\text { say }) \\
& \frac{A_{1}-A_{1}^{H}}{2}=\frac{1}{2}\left[\begin{array}{lcc}
2 i & 2+2 i & -4-6 i \\
-2+2 i & 4 i & 2 \\
4-6 i & -2 & 0
\end{array}\right]=\left[\begin{array}{ccc}
i & 1+i & -2-3 i \\
-1+i & 2 i & 1 \\
2-3 i & -1 & 0
\end{array}\right]=S_{1}(\text { say })
\end{aligned}
$$




$$
\begin{aligned}
& \frac{A_{2}+A_{2}^{H}}{2}=\frac{1}{2}\left[\begin{array}{lcc}
2 & 4 i & -2 i \\
-4 i & -2 & 2 \\
2 i & 2 & 4
\end{array}\right]=\left[\begin{array}{lcc}
1 & 2 i & -i \\
-2 i & -1 & 1 \\
i & 1 & 2
\end{array}\right]=H_{2}(\text { say }) \\
& \frac{A_{2}-A_{2}^{H}}{2}=\frac{1}{2}\left[\begin{array}{lcc}
2 i & -4+2 i & 6+4 i \\
4+2 i & 10 i & 4-8 i \\
-6+4 i & -4-8 i & 0+26 i
\end{array}\right]=\left[\begin{array}{lcc}
i & -2+i & 3+2 i \\
2+i & 5 i & 2-4 i \\
-3+2 i & -2-4 i & 13 i
\end{array}\right]=S_{2}(\text { say }) \\
& A_{B}=\left[\begin{array}{lcc}
2 & 1-3 i & -3+2 i \\
1+3 i & 1 & i \\
-3-2 i & -i & 2
\end{array}\right] \cup\left[\begin{array}{lcc}
1 & 2 i & -i \\
-2 i & -1 & 1 \\
i & 1 & 2
\end{array}\right]+ \\
& {\left[\begin{array}{lcc}
i & 1+i & -2-3 i \\
-1+i & 2 i & 1 \\
2-3 i & -1 & 0
\end{array}\right] \cup\left[\begin{array}{lcc}
i & -2+i & 3+2 i \\
2+i & 5 i & 2-4 i \\
-3+2 i & -2-4 i & 13 i
\end{array}\right]}
\end{aligned}
$$

Hence, $A_{B}=\mathrm{H}_{\mathrm{B}}+\mathrm{S}_{\mathrm{B}}$ (Say), where $\mathrm{H}_{\mathrm{B}}$ is bihermitian and $\mathrm{S}_{\mathrm{B}}$ is skew bihermitian matrices.

\section{Conclusion}

Some of the properties of hermitian matrices are proved for bihermitian matrices. In a similar way all the properties of hermitian matrices can be verified for bihermitian matrices.

\section{References}

[1] Frank Ayres. JR, Theory and Problems of Matrices, Schaum's outline series, SI (Metric Ed.),P.No.10-19

[2] Richard Bronson, Matrix Methods: An Introduction(II Ed.),P.No.422-427

[3] W.B.Vasantha Kandasamy,Florentin Samarandache,K.Ilanthendral,Introduction to bimatrices,2005.

[4] W.B.Vasantha Kandasamy,Florentin Samarandache,K.Ilanthendral ,Applications of bimatrices to some Fuzzy and Neutrosophic models,Hexis.Phoenix,Arizone,2005. 